\title{
Alcohol Schema Acquisition in Preschoolers: Differences Between Children of Alcoholics and Children of Nonalcoholics
}

\author{
Robert A. Zucker, Stephen B. Kincaid, Hiram E. Fitzgerald, and C. Raymond Bingham
}

\begin{abstract}
Cognitive schemas provide the structure within which children organize their knowledge and beliefs about the use of alcohol. The development of schemas about alcohol should be affected both by age and parental patterns of alcohol use. We examined differences in alcohol schema development among 139 male children of alcoholics (COAs) and 82 controls [children of nonalcoholics (NCOAs)] utilizing the Appropriate Beverage Task as an indicator of these processes. Overall, the vast majority of the sample identified at least one alcoholic beverage from photographs, even at age 3. COAs were more likely to identify at least one alcoholic beverage. With age controlled, COAs were better able to identify specific alcoholic beverages and correctly identified a larger number of alcoholic beverages. There was a trend for these children of alcoholic men to attribute more alcohollc beverage use to male adults than NCOAs. Moreover, differences in these children's attributions of alcoholic consummatory behavior were predicted by their parents' current consumption levels. Results provide evidence that alcohol schemas are detectable in early childhood and are more common in children from alcoholic homes. Discussion focuses on the potential relevance of these risk attributes to the development of more fully formed alcohol expectancies and to the later emergence of alcohol-related difficulty.

Key Words: Children of Alcoholics, Cognitive Schemas, Family
\end{abstract} Effects, Socialization of Alcohol Expectancies, Risk for Alcoholism.

$\mathbf{A}^{\mathrm{L}}$ LCOHOL ABUSE and alcohol dependence are the most common of all DSM-III-R disorders for men, ${ }^{1,2}$ but the fully developed clinical disorder reflects an endpoint phenomenon of a kind, for which there are likely to be earlier precursive markers. Within this context, survey data from 1992 indicate that nearly $50 \%$ of 16 -year-olds have already gotten drunk at least once. ${ }^{3}$ The same data base indicates that the process of alcohol involvement has already begun for a small subset of 10 -year-olds (i.e., 4th graders), where $7.6 \%$ have used alcohol at least once and $1.3 \%$ already report a first experience of drunkenness. Moreover, the rapid increase in level of alcohol-related symptomatology during adolescence suggests that precur-

From the Departments of Psychiatry and Psychology (R.A.Z., S.B.K), the University of Michigan, Ann Arbor, Michigan, and the Department of Psychology (H.E.F., C.R.B.), Michigan State University, East Lansing, Michigan.

Received for publication November 16, 1994; accepted February 13, 1995

This study was supported by Grant RO1 AA07065 from the National Institute on Alcohol Abuse and Alcoholism. Portions of this work were presented at the 1994 Annual Meeting of the American Psychological Association, Los Angeles, California.

Reprint requests: Robert A. Zucker, Ph.D., University of Michigan Alcohol Research Center, 400 East Eisenhower Parkway, Suite 2A, Ann Arbor, Michigan.

Copyright $(1) 1995$ by The Research Society on Alcoholism.

Alcohol Clin Exp Res, Vol 19, No 4, 1995: pp 1011-1017 sive characteristics may already be in operation, and that the presence of this risk structure is what drives the apparently rapid emergence of adolescent trouble. ${ }^{4}$

One domain of such risk is nonspecific to alcohol use and involves temperament variation, ${ }^{5}$ one is specific to alcohol use and involves variation in appetitive and consummatory effects, ${ }^{6-8}$ and a third is cognitive and motivational. ${ }^{9,10}$ The alcohol dependence syndrome involves both alcohol-seeking and alcohol-using behavior. For drug-seeking to take place, one must be aware that the drug exists, and that it has special reinforcing properties that are likely to be activated if the drug is sought out and consumed. Although the existing literature indicates that a significant part of such learning involves subjective experiences occurring once the drug has been ingested, an emerging literature also indicates that the process of identifying alcohol as a drug, and denoting it as a type of substance with specific reinforcing properties, begins earlier. Preadolescents, and even preschoolers, are known to have some understanding of the contextual, motivational, and normative aspects of alcohol usage. ${ }^{11-18}$

Elsewhere, we have proposed the concept of cognitive schema as the superordinate structure within which children organize their knowledge about patterns of alcohol use, expectancies about results of such use, and attitudes and intentions about their own eventual use.,19 Families are a primary source of socialization regarding attitudes and behaviors of health, ${ }^{20}$ and it is reasonable to expect that exposure to an alcoholic environment would be associated with the precocious development of schemas regarding alcohol. Previous research has associated parental alcohol use patterns with both attitudes and expectancies concerning alcohol in adolescence, ${ }^{17,21,22}$ and alcohol expectancies have also been related to individual differences in actual drinking behavior. ${ }^{23-26}$ Thus, evidence in early childhood that schemas about alcohol are present may also be an early marker of heightened risk for later alcohol abuse or dependence. ${ }^{27}$

Earlier work by our group ${ }^{28-30}$ found that preschool children in the general population already know two of the core alcohol use-schemas of the larger culture: they attribute alcoholic beverage consumption more to adults than children, and more to adult males than adult females. These data indicated that children already understand the 
basic rule structure about adult alcohol use. As a part of that work, a pilot study with a very small sample of alcoholic families found only trend level differences attributable to living in an alcoholic home. ${ }^{31}$ In the present study, using a much larger $n$, and a sample involving children from both alcoholic and nonalcoholic families, we expected to replicate the earlier findings that children already know the core use schemas in the culture. Thus, we anticipated that the ability to identify visual stimuli of alcoholic beverages would vary with exposure, and thus would differ (1) as a function of age (i.e., older children would do better at this than younger children), and (2) as a function of differences in level of alcohol use in the family environment. (3) We also expected that attributions of alcoholic beverage use would differ as a function of exposure to an alcoholic home environment. Those reared in alcoholic homes would be more likely to attribute alcoholic beverage use to social situations where beverages were being consumed.

\section{METHODS}

\section{Sample}

The participants in this study were 223 male children under age 7 (mean age $=4.5$ years, ranging from 2.9 to 6.5 years) in Wave One of the Michigan State University-University of Michigan (MSU-UM) Longitudinal Study ${ }^{32}$ who had data available on alcohol schema development. The MSU-UM study is an ongoing, community-based prospective study of family health and child development that is following a sample of alcoholic men and their families, along with a contrast group of families in which neither parent is a substance abuser.

Participant families were recruited through two methods. First, families with an alcoholic father were recruited through a court network that provided access to all males convicted of driving under the influence within a four-county area of mid-Michigan. To be considered for the project, these men were required to have had a blood alcohol concentration $(\mathrm{BAC})$ of $0.15 \%(150 \mathrm{mg} / 100 \mathrm{ml})$ or higher at first arrest, or a BAC of $0.12 \%$ with a history of multiple alcohol-related arrests. An initial positive alcoholism diagnosis was established through the Short Michigan Alcohol Screening Test ${ }^{33}$ shortly after recruitment, and was subsequently confirmed through the Diagnostic Interview Schedule ${ }^{34}$ to verify that these men met Feighner "probable" or "definite" diagnostic criteria for alcoholism. ${ }^{35}$ Ninety-two percent of the fathers recruited in this manner met the definite criterion level, and $71 \%$ also received a DSM-III-R diagnosis of moderate or severe dependence. In addition, potential participants were required to have a son between 3 and 5 years of age at time of recruitment (referred to as the "target child"); both the father and the target child were required to be residing with the child's biological mother at the time of recruitment, and both mother and father had to provide informed consent. Assent was also obtained from all child participants. Of the total number of men who met initial selection criteria, $79 \%$ agreed to allow their names to be released by the court for contact by study staff. Of these, $92 \%$ of the contacted families agreed to participate. This group is referred to as overt alcoholics.

Mother's drinking status was assessed but was left free to vary and in fact ranged from alcohol dependence to current abstention. The larger study design was set up with different inclusionary criteria for mothers, because female alcoholism is so much less prevalent in the general population. Thus, had we attempted to systematically sample equal numbers of families with and without an alcoholic mother, the recruitment process would have taken three to four times as long, and with concomitant add-on study costs. An even more complete design would sample a subset of families with alcoholic mothers but not fathers. This alternative was also considered, but abandoned because of cost considerations. Nonetheless, the larger study is systematically exploring the manner in which maternal drinking does or does not interface with paternal drinking, and this issue is also considered in the present work.

A second set of families was recruited as a contrast group through door-to-door canvassing of neighborhoods in the same census tract as families from the overt alcoholic group; this manner of recruitment attempted to minimize differences in socioeconomic status. Further demographic matching was based on the target child's gender, age ( \pm 6 months of the overt alcoholic group), and sibling constellation. Through this procedure, a sample of control families was obtained with parents who did not meet Feighner criteria for alcoholism or other drug abuse/ dependence, and who were demographically similar to overt alcoholic families. However, this canvassing procedure sometimes serendipitously yielded families with an alcoholic father who met Feighner criteria, but had no alcohol-related or drug-related arrests during the lifetime of the target child. This latter set of families was also included in the alcoholic group and is referred to as covert alcoholics.

All families included in the study were Caucasian. Given the welldocumented relationship between ethnic/racial status and patterns of drug/alcohol involvement, the limited composition of ethnic/racial minorities in the population from which we were recruiting $(<10 \%)$ and the inability to conduct analyses of such differences with adequate power, it was decided to exclude this variability through sample restriction rather than have it contribute to error variance. All families received some monetary compensation for taking part in the study.

\section{Determining Risk Status}

Risk status was determined by the presence or absence of alcoholism in the family. Children of alcoholics (COAs) were defined by the presence of at least the father meeting the recruitment and diagnostic criteria for alcoholism. For children of nonalcoholics (NCOAs), neither parent made an alcohol or other drug diagnosis.

\section{Assessing Cognitive Schemas Regarding Alcohol Use}

As part of a battery of developmental assessments tracking the emergence of cognitive schemas, a modified version of the Appropriate Beverage Task $^{36}$ was administered to examine the degree to which children understand the normative use structure for alcoholic beverage consumption. All protocols were conducted by experienced child clinicians who were able to establish and maintain rapport effectively even with young and/or unruly children.

Ten photographic stimuli of beverages ( 5 alcoholic, 5 nonalcoholic) were laid out on a table in front of the participant in a random order; 10 drawings of adults and/or children in various contexts were then presented one at a time, also in a random order (Table 1). In every drawing, each character is drinking an unidentified beverage. Participants were asked to use the photographs to specify the beverage each person in each drawing was drinking. Thus, an alcoholic beverage attribution score was assigned to each figure, and summarizing across figures could yield an attribution score based on drinker's gender, age, or the context in which the consumption was attributed to take place.

In addition, participants were asked to provide the names for each of the 10 beverage photographs. Of those that could not be recalled, the experimenter would name each in turn and ask the child to match the photographic stimulus with the beverage being named. Thus, scores could vary from 0 (the child was unable to identify any alcoholic beverage correctly) to 5 (the child correctly identified every alcoholic beverage). Two judges (blinded to family risk status) independently coded all data. For interrater reliability, a subset of 20 participants were coded by each, with $99.1 \%$ agreement on coding decisions.

For analytic purposes, a subset of the protocol responses were selected that provided a balanced within-subject matrix nested in a between-groups ANOVA design (COA versus NCOA) that would test the individual and interactive effects of properties of the stimulus cards on the attribution of alcoholic beverages. Within-subject factors were context of drinking (Party versus Nonparty scene), drinker's age (Adult versus Child), and drinker's 
Table 1. Descriptions of Stimulus Cards of the Appropriate Beverage Task

\begin{tabular}{|c|c|c|c|c|c|}
\hline \multirow[b]{3}{*}{ Stimulus } & \multirow[b]{3}{*}{ Description } & \multicolumn{4}{|c|}{ No. of observations } \\
\hline & & \multicolumn{2}{|c|}{ Adults } & \multicolumn{2}{|c|}{ Children } \\
\hline & & Male & Female & Male & Female \\
\hline \multicolumn{6}{|c|}{ Nonparty Context } \\
\hline Card I & A male adult, a female adult, a male child, and a female child are sitting down to a Thanksgiving dinner & 1 & 1 & 1 & 1 \\
\hline Card II & A female adult is sitting alone, watching television & 0 & 1 & 0 & 0 \\
\hline Card III & A male adult and a female adult sitting in front of a fireplace; a fire is burning & 1 & 1 & 0 & 0 \\
\hline Card VI & Two male children are eating lunch together & 0 & 0 & 2 & 0 \\
\hline Card VII & A male adult is sitting alone, watching television & 1 & 0 & 0 & 0 \\
\hline Card VIII & A male adult, a female adult, a male child, and a female child are playing a family baseball game & 1 & 1 & 1 & 1 \\
\hline Card IX & Two female children are eating lunch together & 0 & 0 & 0 & 2 \\
\hline \multicolumn{6}{|l|}{ Party Context } \\
\hline Card IV & Two male adults and two female adults at a New Year's Eve party & 2 & 2 & 0 & 0 \\
\hline Card V & A male adult, a female adult, a male child, and a female child are having a 4th of July picnic & 1 & 1 & 1 & 1 \\
\hline Card $X$ & Two male children and two female children at a birthday party & 0 & 0 & 2 & 2 \\
\hline
\end{tabular}

gender (Male versus Female). Thus, although all data were utilized in analyses pertinent to the child's knowledge of alcoholic beverages, the attribution analyses omitted data from cards I, V, and VIII to provide a balanced design for context, drinker's age, and drinker's gender (i.e., a 2 $\times 2 \times 2$ within-subject ANOVA matrix), with an additional betweensubjects factor (COA status). Two observations were made within each cell (e.g., adult males in a party context). Effects for order of card presentation were tested in early analyses, but were not found to be significant. They are not discussed further in any of the results.

\section{Assessing Current Parental Alcohol Consumption}

Current alcohol consumption was assessed via a questionnaire that incorporates items from the American Drinking Practices Survey, ${ }^{37}$ the 1978 National Institute on Drug Abuse High School Survey, ${ }^{38}$ and from the V.A. Medical Center Research Questionnaire. ${ }^{39}$ The version used herein ${ }^{40}$ contained information necessary to code a revision of the Quantity-Frequency-Variability (QFV) Index ${ }^{37}$ of drinking level called QFV$\mathrm{R}^{41}$ This measure uses the basic Cahalan et al. scoring system, but rather than combining the Quantity-Variability (QV) classification with the Frequency classification to yield a 5-category classification, the score is obtained by multiplying the QV class $\times$ the approximate number of drinking episodes/year (based on the reported average frequency). This yields a $0-21,000$ score that is then subjected to a logarithmic transformation (base 10). This revision of the index increases the sensitivity of the measure, given that so many of the alcoholic men in the current sample would be classified as heavy drinkers.

\section{RESULTS}

\section{Demographic Characteristics of the Sample}

Of the 223 male children, 139 (62\%) were COAs and 84 $(38 \%)$ were NCOAs. Although age did not vary with risk status $[F(1,221)=0.05, p<0.83]$, differences were found in family socioeconomic status $[F(1,218)=9.06, p<0.01]$ COAs were from lower socioeconomic status families. This effect has been identified in a variety of earlier studies and is simply replicated herein. ${ }^{42}$

\section{Knowledge of Alcoholic Beverage Visual Stimuli}

Overall, $88 \%$ of the sample was able to identify correctly at least one alcoholic beverage. Even among 3-year-olds, the vast majority were already able to identify at least one alcoholic beverage (Table 2). Overall, COAs were significantly more likely than NCOAs to be able to identify at
Table 2. Age Variations in the Ability to Identify Correctly Alcoholic Beverage Stimuli

\begin{tabular}{|c|c|c|c|c|c|}
\hline \multirow{2}{*}{$\begin{array}{c}\text { Correlations } \\
\text { Alcoholic stimulus type }\end{array}$} & \multicolumn{4}{|c|}{$\begin{array}{l}\text { Percentage correctly } \\
\text { identified by age }\end{array}$} & \multirow[b]{2}{*}{$r$} \\
\hline & 3 & 4 & 5 & 6 & \\
\hline Identification of any stimulus & 73.0 & 88.5 & 87.3 & 97.7 & $0.18^{\star \star}$ \\
\hline Beer & 65.7 & 85.3 & 82.3 & 97.7 & $0.23^{* \star *}$ \\
\hline Wine & 34.3 & 41.9 & 66.1 & 65.9 & $0.24^{\star \star \star *}$ \\
\hline Whiskey & 17.1 & 20.0 & 37.1 & 34.9 & $0.15^{\star}$ \\
\hline Gin/vodka/rum & 2.9 & 20.0 & 32.3 & 43.2 & $0.31^{* * *}$ \\
\hline Sherry & 20.0 & 21.3 & 22.6 & 27.3 & 0.05 \\
\hline Mean total no. identified & 1.40 & 1.86 & 2.40 & 2.66 & $0.32^{\star \star \star}$ \\
\hline
\end{tabular}

Note: $n=37$ for age $3, n=78$ for age $4, n=63$ for age 5 , and $n=45$ for age 6. ${ }^{*} p<0.05 ;{ }^{* *} p<0.01 ;{ }^{* * *} p<0.001$.

least one alcoholic beverage (percentage correctly identifying at least one alcoholic beverage: $\mathrm{COAs}=93 \%$, NCOAs $=77 \% ; \chi^{2}=7.75,1 d f, p<0.01$ ), by a factor of 3.2:1. These differences also are apparent at earlier ages; even at age 3, COAs were able to identify correctly at least one alcoholic beverage at a 7.0:1.0 rate, compared with NCOAs $\left(\chi^{2}=5.40,1 d f, p<0.05\right)$, and there was a trend for 3-year-old COAs to be able to identify correctly more alcoholic beverages than 3-year-old NCOAs [COA mean $=$ 1.70$, NCOA mean $=1.00 ; F(1,33)=3.62, p<0.07]$.

COAs were generally better able to identify correctly specific alcoholic beverage stimuli (Fig. 1). This effect was strongest for what is likely the beverage of greatest exposure: beer. This is of particular significance, given no observed incremental difference in the ability to identify nonalcoholic beverages [COA mean $=4.68$, NCOA mean $=$ 4.69; $F(1,215)=0.07, p<0.80]$. Differences between COAs and NCOAs in the ability to identify beer can be ascertained as early as age $3\left(\chi^{2}=7.70, p<0.01\right)$, although these differences are no longer present by age $6\left(\chi^{2}=1.42\right.$, $p<0.23$ ). In addition, across all ages, COAs were able to identify correctly more of the alcoholic beverages [COA mean $=2.25$, NCOA mean $=1.85 ; F(1,214)=5.13, p<$ $0.03]$.

\section{Differential Attributions of Alcoholic Beverage Use}

In addition to being able to identify the alcoholic beverage stimuli, the children differentially assigned alcoholic 


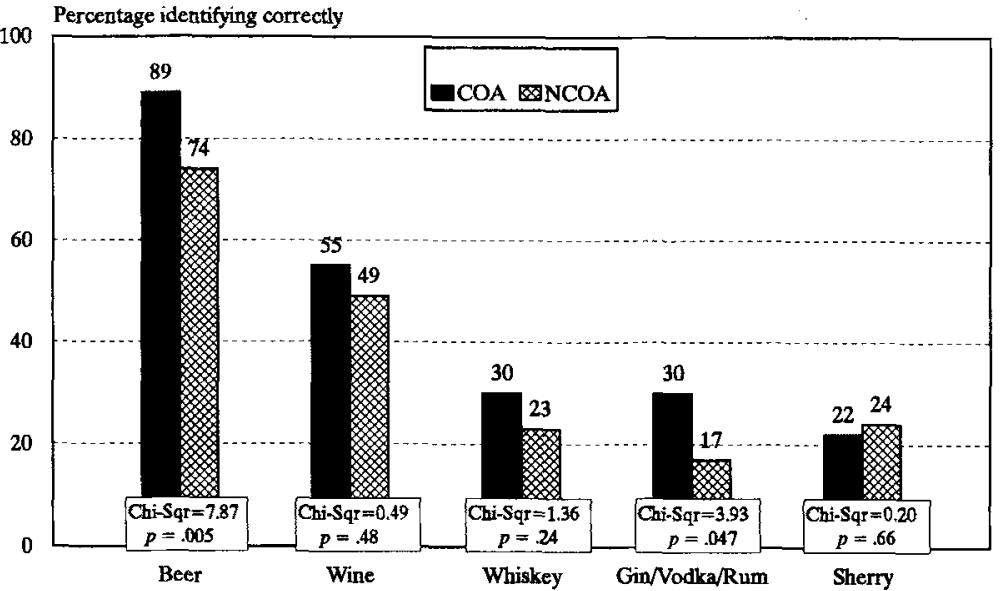

Fig. 1. Knowledge of specific alcoholic beverages among preschooler COAs and NCOAs.
Table 3. Attributions of Alcoholic Beverage Use: ANOVA of Effects of Child's Age, Risk Status, and Drinker's Age and Gender

\begin{tabular}{lcc}
\hline \multicolumn{1}{c}{ Effect properties } & $F$ & $\rho$ \\
\hline Child's age & 4.54 & 0.030 \\
Child's risk status (COA vs. NCOA) & 0.95 & 0.334 \\
& & \\
Drinker's age (adult vs. child) & 0.36 & 0.550 \\
Drinker's gender (male vs. female) & 3.11 & 0.080 \\
Drinking context (party vs. nonparty) & 1.66 & 0.199 \\
& & \\
Drinker's age $\times$ child's age & 9.91 & 0.002 \\
Drinker's age $\times$ child's risk status & 4.17 & 0.043 \\
& & \\
Drinker's gender $\times$ child's age & 8.83 & 0.004 \\
Drinker's gender $\times$ child's risk status & 0.94 & 0.334 \\
& & \\
Drinking context $\times$ child's age & 1.19 & 0.276 \\
Drinking context $\times$ child's risk status & 0.06 & 0.813 \\
Drinker's age $\times$ drinker's gender $\times$ & 2.82 & 0.095 \\
$\quad$ child's risk status
\end{tabular}

Note: Degrees of freedom for all analyses are $(1,218)$. No additional three-way or four-way significant interactions or trends were found.

beverage use to the drinkers depicted on the stimulus cards. We tested for attributional effects using the ANOVA design described previously. A significant main effect was found for the total number of attributions of alcoholic beverages made based on the child's age (after being converted to a categorical variable), and a trend was found based on drinker's gender (Table 3 ). Older children attributed more alcoholic beverage use to drinkers, and more alcoholic beverage use was assigned to male drinkers. No significant main effects existed as a function of COA status or contextual properties of the stimulus cards. Two-way interaction effects were found for drinker's age and child's risk status (compared with NCOAs, COAs attributed more alcoholic beverages to adults by a factor of 1.2 , whereas attributions to children were virtually identical), drinker's age and child's age (the number of attributions made to adults increased $51 \%$ in a linear manner with increasing age of the child, from a mean of 1.7 at age 3 to a mean of 2.8 at age 6 , out of 8 stimulus opportunities; attributions made to children declined $42 \%$ in a linear manner with increasing age of the child, from a mean of 1.0 at age 3 to a mean of 0.5 at age 6 , out of 8 stimulus opportunities), and drinker's gender and child's age (the number of attributions made to males increased $53 \%$ in a linear manner with increasing age of the child, from a mean of 1.5 at age 3 to a mean of 2.3 at age 6 , out of 8 stimulus opportunities; attributions made to females did not vary by $>15 \%$ in either direction, from a mean of 1.3 with increasing age of the child). A trend for a three-way interaction was also found between risk status and the drinker's age and gender. Given that this area has been virtually unexplored to date, and given that the data fell in a direction that would be anticipated on the basis of theory [i.e., that children living with an adult male (the father) who heavily models the use of alcohol would be anticipated to incorporate this use pattern into their own schema structure about "adult men in general"], we felt it was legitimate to conduct univariate analyses of this effect even though it was only a trend. The univariate ANOVA were conducted between risk status groups for each drinker's age and gender pairing. In these analyses, a trend was also found for COAs to attribute more alcoholic beverages to male adults than NCOAs (for means and probabilities, see Fig. 2).

\section{Determinants of Attribution of Alcoholic Beverage Use}

Overall, the children in the study attributed alcoholic beverage use to adult males at almost double the rate they attributed to adult females and approximately four times the rate they attributed to either male or female children (Fig. 2). Table 4 describes the hierarchical regression equations used to predict these individual differences. Given the positive correlation between age and the ability to identify correctly specific beverages, and utilizing the principle that overarching developmental attributes (such as age) are the most parsimonious explanations for behavioral manifestations at any life stage, age was entered as step 1 in these equations. We followed in step 2 with an index of proximal exposure, the QFV-R of the same gender parent. Thus, in predicting attributions of alcoholic beverage consumption to adult male figures, the father's alcohol consumption was 


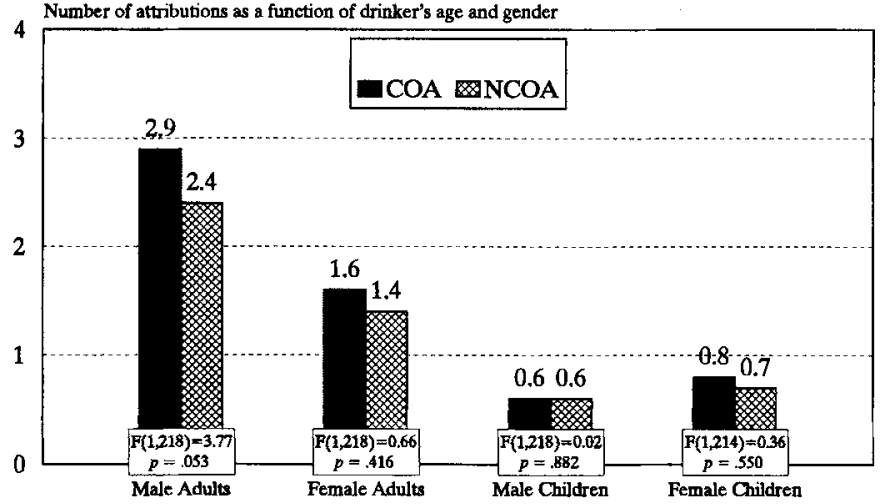

Fig. 2. Attribution of alcoholic beverage use among preschooler COAs and NCOAs.

entered here, and in predicting these attributions to female figures, mother's alcohol consumption was used. The potential additional contribution of exposure to the other parent's consumption was examined by entering that parent's QFV-R in step 3. Only age and father's consumption were significant contributors to the boys' attributions of adult male consumption. Overall, the model accounted for $16 \%$ of the variance, and father's consumption level was by far the strongest factor. Variations in attribution of alcohol use to adult women were predicted only by level of mother's consumption; father's consumption did not play a role. Although the model is significant, the amount of variance accounted for is low (4\%). Clearly, other factors not assessed herein play more of a role.

\section{DISCUSSION}

Alcoholic beverages, as a category of objects in the physical environment, are distinguishable from other categories of substances, because alcohol is the drug of most common use and abuse in adulthood. Alcohol use and abuse has been heavily studied at adolescence, when alcohol-related problems first become obvious. However, a developmental perspective on such phenomena would anticipate that, before the obvious expression of such behavior, there are precursive indicators. Within the context of a sample comprised of a set of families with an alcoholic father and a set of ecologically comparable but nonalcoholic control families, the present study expanded upon previous work using preschoolers with nonclinical parents, and examined early childhood knowledge and attributions about alcohol as indicators of emerging cognitive schemas regarding alcoholic beverages. We found that independent of risk status, the majority of children were able to identify correctly a variety of alcoholic beverages presented by way of photographic stimuli. In other words, at least at a labeling level, knowledge about alcohol and the ability to differentiate alcoholic from nonalcoholic beverages is already present in early childhood. Even at age 3, the majority of children could identify at least one alcoholic beverage. As would be anticipated, the ability to identify specific alcoholic beverages and the breadth of knowledge about alcohol both increased with age. In addition, these results replicated our earlier findings in that children attributed alcoholic beverage use to adults more than children, and to adult men more than adult women. Data indicate that these patterns become more sharply articulated as the child grows older; they also document the extent to which the cultural rule structure about alcohol use permeates the early childhood environment. Although this structure becomes increasingly internalized as the child ages (and increases his opportunities for exposure to it), it is detectable even before entry to school.

Rearing environment differences in alcohol schema presence also were found: COAs were better able to identify at least one alcoholic beverage, were better able to identify specific alcoholic beverages, and were able to identify a larger number of alcoholic beverages. COAs were more likely to attribute alcoholic beverages to adults rather than children, and there are further indications that COAs already understood that adult males were more likely to be consumers of alcohol than any other age-gender combination. The regression analyses also indicated that the attributions are reflective of variations in the same gender parent's consumption level. Results thus strongly suggest that these emerging schemas are shaped by learning experiences occurring within the home. These findings are, to our knowledge, the first reported about young children that indicate that their capacity to characterize their parents' choice of drug of abuse (i.e., alcoholic beverages) is more effectively learned in high-risk environments, and further, that COAs are more likely to describe this drug as a beverage of appropriate social use among adults. In other words, the presence of a schema for adult use of this drug

Table 4. Predicting Child Attributions of Alcoholic Beverage Use: Hierarchical Regressions of Child's Age and Parental Alcohol Consumption on Child Attributions

\begin{tabular}{|c|c|c|c|c|}
\hline & $R^{2}$ & $R^{2}$ change & $F$ change & $F$ model \\
\hline \multicolumn{5}{|l|}{ Attributions to adult males } \\
\hline Step 1. Child's age & 0.03 & 0.03 & $5.95^{*}$ & $5.95^{\star}$ \\
\hline Step 2. Paternal alcohol consumption & 0.15 & 0.12 & $31.87^{\star \star}$ & $19.31^{\star \star}$ \\
\hline Step 3. Maternal alcohol consumption & 0.16 & 0.01 & 2.75 & $13.90^{\star \star}$ \\
\hline \multicolumn{5}{|l|}{ Attributions to adult females } \\
\hline Step 1. Child's age & 0.01 & 0.01 & 1.56 & 1.56 \\
\hline Step 2. Maternal alcohol consumption & 0.04 & 0.03 & $6.58^{\star}$ & $4.09^{*}$ \\
\hline Step 3. Paternal alcohol consumption & 0.04 & 0.00 & 0.00 & $2.72^{*}$ \\
\hline
\end{tabular}

" $p<0.05 ;{ }^{* *} p<0.001$. 
has already been formed, and it is formed more readily in alcoholic homes.

Within the purview of a cognitive-behavioral, social learning theory of the acquisition of drinking behavior, ${ }^{43}$ precocious knowledge and schema formation may be viewed as two early elements in a matrix of risk that would be anticipated to lead to earlier and heavier alcohol use when alcohol becomes available, provided the nesting environment sustains their presence. The rudimentary schemas about alcohol identified herein are to be regarded as building blocks around which the later, more fully articulated alcohol expectancies of adolescence $e^{17,21,22,44}$ are shaped. Moreover, given the ability to accurately identify and label beer at age three, and the knowledge of the alcohol consumption rule structure that already exists among these preschoolers, these data indicate that if there is a "critical period" for expectancy formation, it occurs substantially earlier than the third grade time line proposed by Miller et al. ${ }^{14}$

We have suggested elsewhere that the presence of such schemas, nested in an environment that sustains their development and encourages the development of nonalcoholspecific risks, is the breeding ground within which the most severe alcohol problems are likely to emerge and then crystalize. ${ }^{4}$ The longitudinal study from which these data were drawn will be tracking these connections as the children and their families grow older. As the study progresses, it will be essential to examine the degree to which early schemas mediate the formation of later positive expectancies about alcohol use, as well as to chart the manner in which other child and family characteristics sustain or dilute the connections as the children grow older.

\section{ACKNOWLEDGMENTS}

We are especially indebted to the families participating in the MSU-UM Longitudinal Study; Susan K. Refior, Field Coordinator for the study; Roger E. Jansen, who did the bulk of the coding; and Robert B. Noll, who played a significant early role in planning and supervising the protocol from which these data were derived. We also thank two anonymous reviewers whose comments substantially improved the quality of this paper.

\section{REFERENCES}

1. American Psychiatric Association: Diagnostic and Statistical Manual of Mental Disorders, ed 3-rev. Washington, D.C., American Psychiatric Association, 1987

2. Robins LN, Regier DA: Psychiatric Disorders in America: The Epidemiologic Catchment Area Study. New York, Free Press, 1991

3. Johnson LD, O'Malley PM, Bachman JG: National Survey Results on Drug Use from the Monitoring the Future Study, 1975-1992. Volume 1: Secondary School Students. Rockville, MD, National Institute on Drug Abuse, 1993

4. Zucker RA, Fitzgerald HE, Moses HD: Emergence of alcohol problems and the several alcoholisms: A developmental perspective on etiologic theory and life course trajectory, in Cicchetti D, Cohen DJ (eds): Manual of Developmental Psychopathology, vol 2. New York, Wiley, 1995

5. Tarter RE, Vanyukov M: Alcoholism: A developmental disorder. J Consult Clin Psychol 62:1096-1107, 1994

6. Sayette MA: An appraisal-disruption model of alcohol's effects on stress responses in social drinkers. Psychol Bull 114:459-476, 1993

7. Newlin, D: Alcohol challenge in high risk individuals, in Zucker RA, Boyd GM, Howard J (eds): The Development of Alcohol Problems: Exploring the Biopsychosocial Matrix of Risk. Rockville, MD, National Institute on Alcohol Abuse and Alcoholism, Res. Monogr. 26, 1994, pp $47-68$

8. Thomasson HR, Edenberg HJ, Crabb DW, Mai X-L, Jerome RE, Li T-K, Wang S-P, Lin Y-T, Lu R-B, Yin S-J: Alcohol and aldehyde dehydrogenase genotypes and alcoholism in Chinese men. Am J Hum Genet 48:677-681, 1991

9. Goldman MS, Brown SA, Christiansen BA, Smith GT: Alcoholism and memory: Broadening the scope of alcohol expectancy research. Psychol Bull 11:137-146, 1991

10. Petraitis J, Flay BR, Miller TQ: Reviewing theories of adolescent substance abuse: Organizing pieces in the puzzle. Psychol Bull 117:67-86, 1995

11. Casswell S, Gilmore LL, Silva P, Brasch P: What children know about alcohol and how they know it. Br J Addict 83:223-227, 1988

12. Gaines LS, Brooks PH, Maisto S, Dietrich M, Shagena M: The development of children's knowledge of alcohol and the role of drinking. J Appl Dev Psychol 7:441-457, 1989

13. Jahoda G, Davies JB, Tagg S: Parents' alcohol consumption and children's knowledge of drinking and usage patterns. Br J Addict 74:297303, 1980

14. Miller PM, Smith GT, Goldman MS: Emergence of alcohol expectancies in childhood: A possible critical period. J Stud Alcohol 53:343-349, 1990

15. Noll RB, Zucker RA, Greenberg GS: Identification of alcohol by smell among preschoolers: Evidence for early socialization about drugs occurring in the home. Child Dev 61:1520-1527, 1990

16. Spiegler DL: Children's attitudes toward alcohol. J Stud Alcohol 44:545-552, 1983

17. Zucker RA, Noll RB: The interaction of child and environment in the early development of drug involvement: $A$ far ranging review and a planned very early intervention. Drugs Society 2:57-97

18. Fossey E: Identification of alcohol by smell among young children: An objective measure of early learning in the home. Drug Alcohol Depend 34:29-35, 1993

19. Zucker RA, Fitzgerald HE, Noll RB: The development of cognitive schemas about drugs among preschoolers. Paper presented at The Socialization of Drinking in Children Symposium conducted at the annual meeting of the Society for Research in Child Development, Seattle, WA, April 1991

20. Tinsley BJ: Multiple influences on the acquisition and socialization of children's health attitudes and behavior: An integrative review. Child Dev 63:1043-1069, 1992

21. Brown SA, Creamer VA, Stetson BA: Adolescent alcohol expectancies in relation to personal and parental drinking patterns. $J$ Abnorm Psychol 96:117-121, 1987

22. Christiansen BA, Goldman MS: Alcohol-related expectancies versus demographic background variables in the prediction of adolescent drinking. J Consult Clin Psychol 51:249-257, 1983

23. Christiansen BA, Smith GT, Roehling PV, Goldman MS: Using alcohol expectancies to predict adolescent drinking behavior after one year. J Consult Clin Psychol 57:93-99, 1989

24. Roehling PV, Smith GT, Goldman MS, Christiansen BA: Alcohol expectancies predict drinking: A three-year longitudinal study. Paper presented at the annual meeting of the American Psychological Association, New York, August 1987

25. Smith GT, Roehling PV, Christiansen BA, Goldman MS: Alcohol expectancies versus demographics to predict adolescent drinking longitudinally. Paper presented at the annual meeting of the American Psychological Association, New York, August 1987

26. Southwick L, Steele C, Marlatt GA, Lindell M: Alcohol-related expectancies: Defined by phase of intoxication and drinking experience. J Consult Clin Psychol 49:713-721, 1981

27. Zucker RA, Fitzgerald HE: Early developmental factors and risk 
for alcohol problems. Alcohol Health Res World 15:18-24, 1991

28. Noll RB, Zucker RA, Weil C, Greenberg GS: Cognitive factors in substance abuse: The case for early learning. Pharmacol Biochem Behav 20:989, 1984 (abstr)

29. Greenberg GS, Zucker RA, Noll RB: The development of cognitive structures about alcoholic beverages among preschoolers. Paper presented at the annual meeting of the American Psychological Association, Los Angeles, CA, August 1985

30. Zucker, RA: The four alcoholisms: A developmental account of the etiologic process, in Rivers PC (ed): Alcohol and Addictive Behaviors: Nebraska Symposium on Motivation, 1986. Lincoln, NE, University of Nebraska Press, 1987

31. Noll RB, Zucker RA: Developmental findings from an alcoholic vulnerability study: The preschool years. Paper presented at the annual meeting of the American Psychological Association, Anaheim, CA, August 1983

32. Zucker RA, Fitzgerald HE: Risk and coping in children of alcoholics: Years 6-10 of the Michigan State University Longitudinal Study. East Lansing, MI, Michigan State University, 1992

33. Selzer ML, Vinokur A, vanRooijen L: A self-administered short Michigan Alcoholism Screening Test (SMAST). J Stud Alcohol 36:117126, 1975

34. Robins LN, Helzer JE, Croughan J, Ratcliff K: National Institute of Mental Health Diagnostic Interview Schedule. Arch Gen Psychiatry 38: 381-389, 1981

35. Feighner J, Robins E, Guze S, Woodruff R, Winokur G, Munoz R: Diagnostic criteria for use in psychiatric research. Arch Gen Psychiatry 26:57-63, 1972

36. Penrose GB: Perceptions of five and six year old children concern- ing cultural drinking norms. Unpublished doctoral dissertation. Berkeley, CA, University of California, 1978

37. Calahan D, Cisin IH, Crossley HM: American Drinking Practices: A National Study of Drinking Behavior and Attitudes. New Brunswick, NJ, Rutgers Center of Alcohol Studies, 1969

38. Johnston LD, Bachman JG, O'Malley PM: Drugs and the Class of '78: Behaviors, Attitudes, and Recent National Trends. Rockville, MD, National Institute on Drug Abuse: Division of Research. U.S. Department of Health, Education, and Welfare, 1979

39. Schuckit MA: Research Questionnaire. Mimeo, Alcoholism Treatment Program. San Diego, CA, V.A. Medical Center, University of California, 1978

40. Zucker RA, Noll RB, Fitzgerald HE: Drinking and Drug Historyrevised edition (version 3). Unpublished questionnaire. East Lansing, MI, Department of Psychology, Michigan State University, 1988

41. Zucker RA, Davies WH: The Revised Quantity-Frequency-Variability Index: Rationale and Formulae. Unpublished manuscript. East Lansing, MI, Department of Psychology, Michigan State University, 1989

42. Fitzgerald HE, Zucker RA: Socioeconomic status and alcoholism: The contextual structure of developmental pathways to addiction, in Fitzgerald HE, Lester BM, Zuckerman B (eds): Children of Poverty: Research, Health, and Policy Issues. New York, Garland 1995, pp 125-148

43. Goldman MS, Brown SA, Christiansen BA: Expectancy theory: Thinking about drinking, in Blane HT, Leonard KE (eds): Psychological Theories of Drinking and Alcoholism. New York, Guilford, 1987, pp $181-226$

44. Smith GT, Goldman MS: Alcohol expectancy theory and the identification of high-risk adolescents. J Res Adoles 4:229-247, 1994 